\title{
ac conductance through an interacting quantum dot
}

\author{
Björn Kubala and Florian Marquardt \\ Physics Department, Arnold Sommerfeld Center for Theoretical Physics, and Center for NanoScience, Ludwig-Maximilians-Universität, \\ 80333 Munich, Germany
}

(Received 21 October 2009; revised manuscript received 9 February 2010; published 15 March 2010)

\begin{abstract}
We investigate the linear ac conductance for tunneling through an arbitrary interacting quantum dot in the presence of a finite dc bias. In analogy to the well-known Meir-Wingreen formula for the dc case, we are able to derive a general formula for the ac conductance. It can be expressed entirely in terms of local correlations on the quantum dot in the form of a Keldysh block diagram with four external legs. We illustrate the use of this formula as a starting point for diagrammatic calculations by considering the ac conductance of the noninteracting resonant-level model and deriving the result for the lowest order of electron-phonon coupling. We show how known results are recovered in the appropriate limits.
\end{abstract}

DOI: 10.1103/PhysRevB.81.115319 PACS number(s): 73.23. $-\mathrm{b}, 71.38 .-\mathrm{k}, 72.10 . \mathrm{Bg}, 72.30 .+\mathrm{q}$

\section{INTRODUCTION}

Recent experimental progress enables the study of the dynamics of electronic processes in mesoscopic structures. In a seminal experiment, Gabelli et al. ${ }^{1}$ measured the ac conductance of a mesoscopic $R C$ circuit. Measuring the in-phase and out-of-phase components of the ac conductance at $\mathrm{GHz}$ frequency, they confirmed theoretical predictions for the coherent transport of noninteracting electrons. ${ }^{2-4}$ General interest in time- or frequency-dependent properties of mesoscopic systems is driven by efforts to measure and manipulate quantum coherent systems as quickly as possible, for instance, for applications in quantum information transmission and processing. An important building block for such experiments may be provided by a coherent single-electron source recently realized. ${ }^{5}$ Furthermore, time- or frequency-dependent measurements can yield additional information about internal time and energy scales that are not accessible via the time-averaged dc current.

A cornerstone for many recent theoretical considerations of the time-independent dc current was laid in the 1992 paper of Meir and Wingreen, ${ }^{6}$ who derived a Landauer-type formula, giving the current through a central interacting region in terms of local Keldysh Green's functions of the central region (and the tunnel coupling and Fermi functions of the leads). In this paper, we extend this approach to the finite-frequency case and derive an expression for the current response to a small ac-voltage excitation, which is applied across the central interacting region in addition to a static dc bias, i.e., the linear ac conductance in a nonequilibrium situation. As in the Meir-Wingreen approach, we derive a formula for the current in terms of "local" objects, but in our case, these are no longer the usual Green's functions, i.e., expectation values of two-operator objects, but contain four electronic operators. The actual evaluation of these central objects may in principle be performed employing various techniques and approximations. The approach lends itself, however, to a combination with a diagrammatic calculation perturbative in the interaction strength ${ }^{7}$ as we demonstrate in an exemplary calculation for electron-phonon coupling.

Before turning to the general approach, we note some previous works that have considered special cases. For van- ishing electron-phonon coupling, our approach reproduces calculations based on the scattering matrix method. ${ }^{2,4,8}$ Those calculations rely on a noninteracting single-particle picture, but can be amended to capture screening by incorporating the effects of induced charges on the internal potentials in a self-consistent manner. The current response to a fixed internal potential as calculated by the scattering method (or by our approach) is then used as an input for these calculations. Another route to finite frequency calculations starts from an expression of the current in Green's function formulation for completely general time dependence of the parameters. ${ }^{9}$ Progress can then again be made for the noninteracting case $^{9,10}$ or employing special approximations to the interacting self-energy and Green's function. ${ }^{11,12}$ Kubo relations naturally link the linear ac conductance to current-current correlators. Indeed, as pointed out in Ref. 13, its real part is related to the asymmetric part of the frequency-dependent current noise for a static dc bias. ${ }^{14}$ Derivations of a general Meir-Wingreen-type formula for the noise, however, have been restricted to the symmetrized noise and, furthermore, consider the noninteracting case ${ }^{15}$ or focus on the zerofrequency limit. ${ }^{16,17}$ Finite-frequency conductance or noise has also been studied in a number of special cases, such as Luttinger liquids ${ }^{18,19}$ and the Kondo regime, ${ }^{20}$ while electron-phonon interaction effects have been investigated by full counting statistics. ${ }^{21-23}$

\section{SETTING UP THE PROBLEM}

\section{A. System}

We consider transport through a central region, for instance a quantum dot, between left and right leads. While electrons in the leads can be considered as noninteracting, within the central region, there might be electron-electron or (as considered in the specific calculations in this paper) electron-phonon interaction. The Hamiltonian of the system can then be written as

$$
\hat{H}=\hat{H}_{\text {central }}+\sum_{\alpha=L / R}\left(\hat{H}_{\text {res }, \alpha}+\hat{H}_{T, \alpha}\right) .
$$

Lead Hamiltonian $\hat{H}_{\text {res, } \alpha}=\Sigma_{k} \epsilon_{k \alpha} \hat{c}_{k \alpha}^{\dagger} \hat{c}_{k \alpha}$ and tunnel Hamiltonian $\hat{H}_{T, \alpha}=\sum_{k, n} V_{k \alpha, n} \hat{c}_{k \alpha}^{\dagger} \hat{d}_{n}+$ c.c. are of the standard form 
and the central part, $\hat{H}_{\text {central }}=\Sigma_{n} \varepsilon_{n} \hat{d}_{n}^{\dagger} \hat{d}_{n}+\hat{H}_{\text {int }}$, may include electron-electron or electron-phonon coupling within $\hat{H}_{\text {int }}$.

We want to consider the nonequilibrium situation, where on top of a finite dc bias, a small ac-excitation voltage $V_{\beta}$ of frequency $\omega_{\mathrm{ac}}$ is applied to lead $\beta$. The resulting finite-frequency current $I_{\alpha}$ flowing from lead $\alpha$ to the central region is given by the linear ac conductance, $I_{\alpha}\left(\omega_{\mathrm{ac}}\right)=\mathcal{G}_{\alpha \beta}\left(\omega_{\mathrm{ac}}\right) V_{\beta}\left(\omega_{\mathrm{ac}}\right)$.

\section{B. Kubo formalism}

Considering linear response with respect to the ac excitation, we can use Kubo formalism, but due to the finite dc bias, the expectation values in the Kubo formalism have to be taken for the nonequilibrium finite-bias state. The standard Kubo approach directly yields the linear conductance $\mathcal{G}_{\alpha \beta}$ in terms of a (retarded) current-current correlator, $\mathcal{G}_{\alpha \beta}\left(\omega_{\mathrm{ac}}\right)=\left[K_{\alpha \beta}\left(\omega_{\mathrm{ac}}\right)-K_{\alpha \beta}(0)\right] /\left(i \omega_{\mathrm{ac}}\right)$, where

$$
K_{\alpha \beta}\left(\omega_{\mathrm{ac}}\right)=-\frac{i}{\hbar} \int_{0}^{\infty} d t e^{i \omega_{\mathrm{ac}} t}\left\langle\left[\hat{I}_{\alpha}(t), \hat{I}_{\beta}(0)\right]\right\rangle .
$$

The current operators are of similar form as the tunnel Hamiltonian

$$
\begin{aligned}
-\frac{1}{(-e)} \hat{I}_{\alpha}(t) & =\dot{\hat{N}}_{\alpha}(t)=\frac{i}{\hbar}\left[\hat{H}, \hat{N}_{\alpha}\right]=\frac{i}{\hbar}\left[\hat{H}_{T, \alpha}, \hat{N}_{\alpha}\right] \\
& =-\frac{i}{\hbar} \sum_{k n}\left(V_{k \alpha, n} \hat{c}_{k \alpha}^{\dagger} \hat{d}_{n}-V_{n, k \alpha} \hat{d}_{n}^{\dagger} \hat{c}_{k \alpha}\right) .
\end{aligned}
$$

\section{KELDYSH APPROACH TO THE AC CONDUCTANCE}

\section{A. Reminder: Meir-Wingreen approach}

To understand how and to what extent progress can be made in further simplifying the general expression of Eq. (1), it is instructive to recall the procedure for calculating the stationary current for a dc bias only. ${ }^{6}$ In that case, the starting point is the expression for the expectation value (with respect to a stationary nonequilibrium state) of the current operator $\left\langle\hat{I}_{\alpha}\right\rangle$, which is expressed as in Eq. (2) above. This corresponds to a diagram with one external vertex with dot- and lead-electron lines, which are contracted to a dot-lead Green's function. In their famous 1992 paper, ${ }^{6}$ Meir and Wingreen expressed this Green's function by dot-dot Green's functions (fully including all interactions as well as tunneling) and the simple lead-lead Green's function and prefactors from the tunneling amplitudes. Execution of the energy sum [the sum over all lead levels in Eq. (2)] then yields their final expression for the current (Eq. 6 of Ref. 6), where the various Keldysh dot-dot Green's functions (i.e., $G^{R / A / K}$ ) enter with different prefactors containing the coupling strengths and Fermi functions of the leads. The problem of calculating the current involving dot-lead Green's functions is thus reduced to the calculation of dot-electron Green's functions only (which, of course, still involve the effects of interaction and tunneling to the leads).
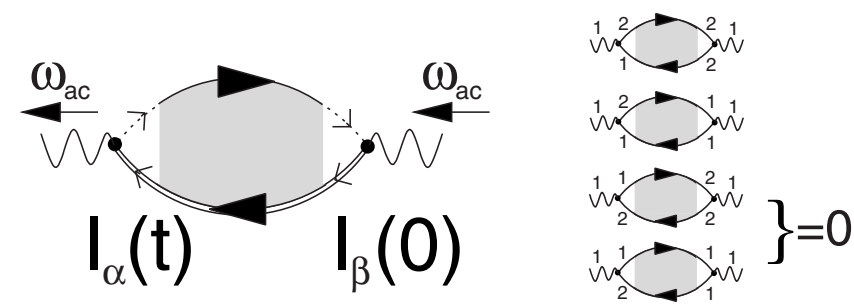

FIG. 1. Correlator needed for the ac conductance. One leadelectron line (dotted line) and one dot-electron line (double line) are connected to each outer vertex. The two contributions in the current vertex, Eq. (2), corresponding to an in- or outgoing dot-electron line (and vice versa for the lead electron) yield different signs for the various configurations. The four diagrams at the right show the possible combinations of Keldysh indices at the inner legs, with the lower two vanishing. A single solid line stands for either dotelectron or lead-electron lines.

In this paper, we perform the equivalent step for the linear ac conductance. As seen above, the calculation starts from the current-current correlator, an object with four dot- and lead-electron operators, which can be reduced to the calculation of a four dot-electron operator object. It corresponds to the central part of a loop diagram with four outer legs (see Fig. 2). In general, this object will not be separable in a product of two Green's functions, but contains lines connecting the upper and lower branches of the loop. In the MeirWingreen formula for the current (Eq. 6 of Ref. 6), the three Green's functions $G^{R / A / K}$ appearing correspond to all possible combination of Keldysh indices 1 and 2 at beginning and end of the dot-electron's line in a diagrammatic representation [we use the following matrix representation of the nonequilibrium Green's functions $\left(\begin{array}{cc}G^{R} & G^{K} \\ 0 & G^{A}\end{array}\right)=\left(\begin{array}{cc}G^{11} & G^{12} \\ G^{21} & G^{22}\end{array}\right)$, where the Keldysh Green's function component $G^{21}$ vanishes by construction $^{7}$ ]. In a similar manner, we may expect to encounter all 16 possible combinations of Keldysh indices on the four legs. In fact, the final expression we will derive in the following turns out to contain only three different Keldysh-index combinations, which considerably eases the calculational effort.

\section{B. Keldysh structure of the current-current correlator}

The correlator $K_{\alpha \beta}\left(t, t^{\prime}\right)=K_{\alpha \beta}\left(t-t^{\prime}, 0\right)$ has the same structure as a retarded Green's function, but with a pair of creation and destruction operators for each of the current operators at the two time variables (see the schematic diagrammatic representation in Fig. 1). Each current operator appears as an external vertex with one lead-electron line (dotted line) and one dot-electron line (double line); one of them entering, one leaving the vertex. ${ }^{25}$ The two possibilities (for each vertex) carry signs according to Eq. (2). In general, in between the four legs starting from the two vertices, there will be a complicated diagram involving many interaction lines, as indicated by the shaded box in Fig. 1. The simplest type of diagram, however, is encountered in the noninteracting case. Then all operators are directly contracted, resulting in two Green's function running antiparallel (one starts at $t$ going to $t^{\prime}$, the other goes from $t^{\prime}$ to $t$ ). Langreth's theorem 


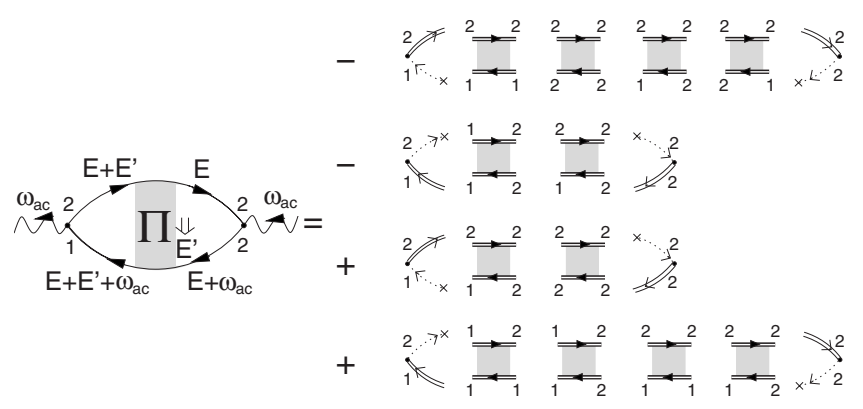

FIG. 2. Each correlator contains four combinations of in or out tunneling at the outer vertices. The dot-lead Green's functions are split into lead-lead and dot-dot Green's functions, so that the correlator can be expressed in terms of a central object $\Pi$ containing dot operators only, represented by gray boxes (with Keldysh indices at the legs indicated). For example, ${ }_{1}^{2} \Pi_{1}^{2}$ occurs in the first and last lines and these diagrams contribute to the term displayed in Eq. (3).

then yields so-called analytical continuation rules, giving the proper combination of nonequilibrium Green's function for the different Keldysh components of the product. $^{7}$ For instance, the object with two antiparallel Green's functions $C\left(t, t^{\prime}\right)=A\left(t, t^{\prime}\right) B\left(t^{\prime}, t\right)$ has the retarded component of $C^{R}\left(t, t^{\prime}\right)=A^{<}\left(t, t^{\prime}\right) B^{A}\left(t^{\prime}, t\right)+A^{R}\left(t, t^{\prime}\right) B^{<}\left(t, t^{\prime}\right)$ $=\frac{1}{2}\left(A^{K} B^{A}+A^{R} B^{K}\right)$. These rules correspond to applying the standard rules for Keldysh indices at the outer vertices. Turning to the interacting case, we use these same rules for the external vertices of the full correlator. We find four possible combinations of Keldysh indices on the inner legs of the correlator loop (see Fig. 1). Note that two of these combinations would include the vanishing Green's function component $G^{21}$ without further interaction lines added to the simple loop diagram. In that case, the Langreth result is directly recovered. In the Appendix, we argue that the two latter index combinations vanish also after including all interaction lines and we are left with only the upper two combinations in Fig. 1.

\section{Eliminating the leads}

Each of the two external vertices is connected to a leadelectron line. Moving away from the vertices along these lines, we will encounter a tunneling event changing the leadelectron line to a dot-electron line. ${ }^{26}$ Note that there can be no interaction line in between the external vertex and the first tunneling, as interaction is only present within the dot region. The Green's function corresponding to the considered line is now split into a product of a lead-lead Green's function, a tunneling matrix element, and a dot-electron Green's function. The Keldysh indexing of those Green's functions follows the rules of matrix multiplication in Keldysh space (see Fig. 2). This split-off procedure has to be performed for the four possible orientations of dot and lead lines at the external vertices [taking account of the proper signs according to Eq. (2)]. We proceed by evaluating the sums over lead states contained in the outer current vertices and in the inner tunneling vertices marked by crosses in Fig. 2. For that, we change from time to frequency or energy domain. We introduce the energy $E$, which encircles the loop, and the energy
$E^{\prime}$ exchanged within the central object between upper and lower lines (see Fig. 2). The summations are then executed under the standard assumption of the wide-band limit to yield $\Sigma_{k}\left|V_{\alpha}\right|^{2} g_{\alpha}^{R / A}=\mp \frac{i}{2} \Gamma_{\alpha}, \quad \Sigma_{k}\left|V_{\alpha}\right|^{2} g_{\alpha}^{K}=-i \Gamma_{\alpha}\left(1-2 f^{\alpha}\right)$, where $g_{\alpha}^{R / A / K}$ are the bare lead Green's functions, $f^{\alpha}$ the Fermidistribution function of lead $\alpha$, and $\Gamma_{\alpha}$ is the coupling strength defined as $\Gamma_{\alpha}=2 \pi \Sigma_{k}\left|V_{\alpha}\right|^{2}$. For ease of notation, we consider a single level in the central region here and in the following. The simple extension to the general case is discussed below.

\section{General Keldysh formula for the ac conductance}

Separating out the leads in that manner, we are left with a central object $\Pi$ with four legs of dot-electron lines, which includes all possible further interaction lines in its central block (see Fig. 2). Starting from the two remaining diagrams of Fig. 1, we can group together all contributions with the same indices on the central object $\Pi$. We find that all but three contributions cancel and the final result for the frequency-dependent retarded current-current correlator can be written as

$$
\begin{aligned}
K_{\alpha \beta}^{C}= & -i \frac{e^{2}}{\hbar} \Gamma_{\alpha} \Gamma_{\beta} \int \frac{d E}{2 \pi} \int \frac{d E^{\prime}}{2 \pi}\left[f_{E}^{\beta}-f_{E+\omega_{\mathrm{ac}}}^{\beta}\right] \\
& \times\left[{ }_{1}^{2} \Pi_{1}^{2}+\left(1-2 f_{E+E^{\prime}+\omega_{\mathrm{ac}}}^{\alpha}\right)_{2}^{2} \Pi_{1}^{2}-\left(1-2 f_{E+E^{\prime}}^{\alpha}\right)_{1}^{1} \Pi_{1}^{2}\right],
\end{aligned}
$$

plus extra terms for the diagonal conductances, stemming from a direct contraction of lead operators of the outer vertices, $\quad K_{\alpha \alpha}^{\text {direct }}=-\frac{e^{2}}{\hbar} \Gamma_{\alpha} \int \frac{d E}{2 \pi}\left[f_{E+\omega_{\mathrm{ac}}}^{\alpha} G_{E}^{A}+f_{E-\omega_{\mathrm{ac}}}^{\alpha} G_{E}^{R}\right]$, so that $K_{\alpha \beta}=K_{\alpha \beta}^{C}+\delta_{\alpha \beta} K_{\alpha \alpha}^{\text {direct. }}$.

This constitutes a general expression for the ac conductance in terms of a central "dot-operator only" object, which is the equivalent to the dot Green's functions in the MeirWingreen result. This central object $\Pi$ is a Keldysh-type diagram with four external legs. Two dot-electron lines enter the diagram and two leave it. We arrange the diagram so that the lines entering are placed at the upper left and lower right corners. Due to energy conservation the energies carried by the four external lines can be parametrized by three parameters $E, E^{\prime}$, and $\omega_{\mathrm{ac}}$ as indicated in Fig. 2. The four external legs carry Keldysh indices as indicated on $\Pi$. The central object $\Pi$ then contains all possible diagrams within these specifications, fully including tunneling and interaction.

If we wish to consider several levels within the central dot region, each dot line carries extra level indices, in general differing at start and end (due to tunneling to the leads and back into a different level). The couplings $\Gamma_{\alpha}$ become matrices in these dot indices and the trace is taken over the whole expression. For instance, the first term of Eq. (3) would change into $\Gamma_{\alpha, i j} \Gamma_{\beta, k l}^{i 2} \Pi_{1 l}^{2 k}$ with summation over all level indices $i, j, k, l$.

\section{APPLICATIONS}

To demonstrate the application of our result, in this section, we will first reproduce the known result of the nonin- 
teracting ac conductance for a single-level quantum dot. We will further show that our result for the ac conductance reduces to the correct dc limit in considering a single-level system coupled to a single phononic mode in lowest nonvanishing order in the electron-phonon coupling strength.

\section{A. Resonant level model}

Without interaction, there are no phononic lines connecting the upper and lower lines of $\Pi$ and energy exchange between these lines is not possible. The superfluous $E^{\prime}$ integral is then cancelled by a $\delta$ function guaranteeing energy conservation. The terms in Eq. (3) with ${ }_{2}^{2} \Pi_{1}^{2}$ and ${ }_{1}^{1} \Pi_{1}^{2}$ vanish (since they would contain $G^{21}=0$ ), while the ${ }_{1}^{2} \Pi_{1}^{2}$ contribution and the direct part immediately yield

$$
\begin{aligned}
\mathcal{G}_{\alpha \beta}^{(0)}= & \frac{e^{2}}{\hbar} \frac{\Gamma_{\alpha} \Gamma_{\beta}}{\omega_{\mathrm{ac}}} \int \frac{d E}{2 \pi}\left(f_{E+\omega_{\mathrm{ac}}}^{\beta}-f_{E}^{\beta}\right) G_{E}^{A, T} G_{E+\omega_{\mathrm{ac}}}^{R, T} \\
& +\delta_{\alpha \beta} \frac{e^{2}}{\hbar} \frac{i \Gamma_{\alpha}}{\omega_{\mathrm{ac}}} \int \frac{d E}{2 \pi}\left(f_{E+\omega_{\mathrm{ac}}}^{\beta}-f_{E}^{\beta}\right)\left(G_{E}^{A, T}-G_{E+\omega_{\mathrm{ac}}}^{R, T}\right) .
\end{aligned}
$$

The noninteracting dot Green's functions including the tunneling self-energy are $G^{R / A, T}(E)=\left[E-\varepsilon \pm i\left(\Gamma_{L}+\Gamma_{R}\right) / 2\right]^{-1}$. We thus recover the result that can also be derived using methods specialized to the noninteracting case, viz., the fully time-dependent Green's function approach of Ref. 9 or scattering matrix theory. ${ }^{4}$
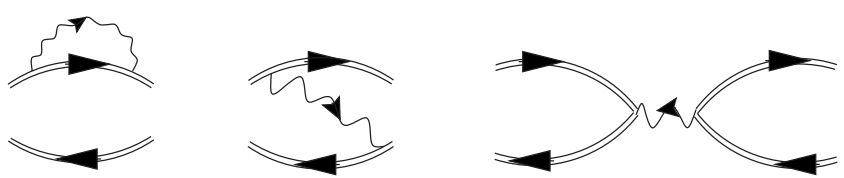

FIG. 3. Three types of second-order diagrams. Diagrams with one Green's function dressed with a phonon line (left), diagrams with one phonon line crossing the loop (middle), and Hartree-type diagrams of two electronic loops coupled by a phononic line (right).

\section{B. Second-order in electron-phonon coupling}

We now consider coupling to phononic modes of frequency $\omega_{\kappa}$, i.e., we take $\hat{H}_{\text {int }}=\sum_{\kappa}\left[g_{\kappa}\left(\hat{a}_{\kappa}+\hat{a}_{\kappa}^{\dagger}\right) \hat{d}^{\dagger} \hat{d}+\hbar \omega_{\kappa} \hat{a}_{\kappa}^{\dagger} \hat{a}_{\kappa}\right]$. We find that in second order in the electron-phonon coupling $g_{\kappa}$, i.e., with one phonon line included in the diagram, there are three different types of contributions. First, we can replace one of the bare dot-electron Green's function by one dressed with a phonon line (see Fig. 3). For the evaluation of this type of diagrams, we accordingly replace bare by dressed Green's function in the zeroth-order result and expand to second order, e.g., $G_{E}^{A, T} \rightarrow G_{E}^{A, T} \sum_{E}^{A, p h} G_{E}^{A, T}$, where the self-energies $\Sigma^{\text {ph }}$ are the lowest-order Fock and Hartree terms, which depend on the bare phonon propagator $D_{\kappa}^{R / A / K}$. Second, there are contributions with one crossing phonon line, which yield

$$
\begin{aligned}
K_{\alpha \beta}^{(2), \mathrm{cross}}= & -i \frac{e^{2}}{\hbar} \Gamma_{\alpha} \Gamma_{\beta} \int \frac{d E}{2 \pi} \int \frac{d E^{\prime}}{2 \pi}\left[f_{E}^{\beta}-f_{E+\omega_{\mathrm{ac}}}^{\beta}\right] i \sum_{\kappa} \frac{g_{\kappa}^{2}}{2} G_{E}^{A, T} G_{E+\omega_{\mathrm{ac}}}^{R, T}\left\{\left[G_{E+E^{\prime}}^{A, T} D_{\kappa, E^{\prime}}^{A} G_{E+E^{\prime}+\omega_{\mathrm{ac}}}^{K, T}+G_{E+E^{\prime}}^{K, T} D_{\kappa, E^{\prime}}^{R} G_{E+E^{\prime}+\omega_{\mathrm{ac}}}^{R, T}\right.\right. \\
& \left.+G_{E+E^{\prime}}^{A, T} D_{\kappa, E^{\prime}}^{K} G_{E+E^{\prime}+\omega_{\mathrm{ac}}}^{R, T}\right]+\left[\left(1-2 f_{E+E^{\prime}+\omega_{\mathrm{ac}}}^{\alpha} G_{E+E^{\prime}}^{A, T} D_{\kappa, E^{\prime}}^{A} G_{E+E^{\prime}+\omega_{\mathrm{ac}}}^{A, T}\right]-\left[\left(1-2 f_{E+E^{\prime}}^{\alpha}\right) G_{E+E^{\prime}}^{R, T} D_{\kappa, E^{\prime}}^{R} G_{E+E^{\prime}+\omega_{\mathrm{ac}}}^{R, T}\right]\right\},
\end{aligned}
$$

where the three terms in the square brackets correspond to the three different indexing variations of $\Pi$ in Eq. (3). The contribution from the third type of diagram in Fig. 3 vanishes in the dc limit in linear response and will not be discussed further here.

The equivalence of the Meir-Wingreen result to the dc limit of our result is most easily seen for the special case of linear response. Then the explicit difference of Fermi functions $f^{\beta}$ in the last two equations results in a derivative, while all other Fermi and Green's functions can be taken at equilibrium and vanishing $\omega_{\mathrm{ac}}$. Contributions from diagrams with one crossing phonon line can then be written

$$
\mathcal{G}_{\alpha \beta}^{(2), \text { cross }}=i \frac{e^{2}}{\hbar} \frac{\Gamma_{\alpha} \Gamma_{\beta}}{\Gamma_{L}+\Gamma_{R}} \int \frac{d E}{2 \pi} f_{E}^{\beta^{\prime}} G_{E}^{R, T}\left[\Sigma_{E}^{R, p h}-\Sigma_{E}^{A, p h}\right] G_{E}^{A} .
$$

Expanding the Green's function in the Meir-Wingreen result to second order in the electron-phonon coupling, we finally recover the proper dc limit. A slightly more involved calculation confirms the equivalence for finite dc bias.

\section{Outlook: Advanced applications}

The application of our technique to investigate decoherence in a quantum-dot Aharonov-Bohm interferometer will be discussed elsewhere. Decoherence effects are also expected to strongly affect the charge relaxation resistance of a mesoscopic $R C$ circuit. ${ }^{24}$ Further possible applications of the technique presented here are numerous: exploration of the combined effects of finite dc bias and small ac excitation, application to different types of interactions, and for different correlated objects, such as finite-frequency noise.

\section{CONCLUSIONS}

We derived a formula for the linear ac conductance, Eq. (3), which is applicable for arbitrary interactions and for a finite applied dc bias. The formula expresses the ac conductance in terms of a central object $\Pi$, which can be calculated using Keldysh diagrammatic methods. We illustrated the application of our formula by explicit calculations for the 
resonant-level model and the case of electron-phonon coupling.

\section{ACKNOWLEDGMENTS}

We acknowledge funding by the DFG via Grants No. SFB/TR 12 and No. SFB 631, NIM, and the Emmy-Noether program and thank $\mathrm{A}$. Clerk for a critical reading of the paper.

\section{APPENDIX: VANISHING INDEX COMBINATIONS}

In constructing the Keldysh diagram representing the retarded current-current correlator, we find that two of four possible combinations of Keldysh indices on the inner legs of the correlator loop (see Fig. 1) lead to the appearance of the vanishing Green's function component $G^{21}$ if we do not allow for interaction lines. In the following, we argue that the two latter index combinations also vanish if we include any number of interaction lines in the diagram. The argument uses only generic features of the Keldysh construction to identify combinations of indices at the legs of a diagram, for which all complicated higher-order diagrams will vanish.

To start with, we consider only one additional interaction line. Then it is easy to see that the same to index combinations vanish, simply by constructing all diagrams according to the indexing rules at the vertices. Vanishing diagrams are then found for two reasons. First, the application of the Keldysh indexing rules at the vertices may lead to the appearance of a line corresponding to the vanishing Green's function component $G^{21}$. Second, diagrams may also vanish due to the fact that the appearance of an advanced or retarded Green's function between two vertices implies a fixed order of the time variables of the two interconnected vertices. Such a time order can lead to a logical contradiction if, for instance, the Green's functions implied that $t_{1}<t_{2}<t_{3}$ and $t_{3}<t_{1}$.

To exploit this property systematically for all higher-order diagrams, we introduce the notion of a time-arrow line, which appears in diagrams indicating a fixed time-ordering of the vertices along this line (see Fig. 4). Such a line runs parallel to $G^{11}=G^{R}$ and antiparallel to $G^{22}=G^{A}$. Analyzing

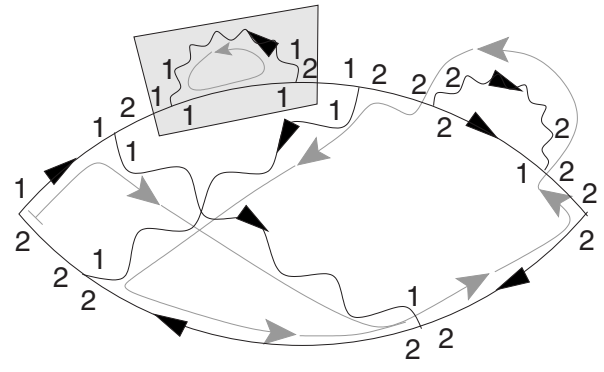

FIG. 4. Time-arrow lines, running parallel to $G^{11}$ or antiparallel to $G^{22}$, do not stop internally, but progress through any possible internal vertex. They may hit themselves, forming a closed loop, which indicates a logical contradiction in the time ordering or end at the external vertices. Additional time-arrow lines (cf. the shaded box) may also exist and enforce vanishing of a diagram if they form a loop.

the 12 different possible index combinations at a vertex (we consider the electron-phonon case here, but the argument is general and relies on the Keldysh indexing rules at the vertices only), we find that if a time-arrow line runs into a vertex, there will always also be such a line leaving the vertex. In consequence, a time-arrow line will never stop at an internal vertex, but will run through a diagram until it hits an outer vertex (where it may end or go on) or until it hits itself and forms a closed loop. Such a closed loop will imply a logical contradiction within the time ordering as discussed above and the corresponding diagram vanishes. Consider now a combination of Keldysh indices at the outer vertices as in the diagram shown in Fig. 4. Due to the vanishing of $G^{21}$, we can infer that two time-arrow lines start at the left external vertex, while at the right vertex, we can have a time-arrow line starting or crossing through the vertex. As the time-arrow lines do not stop internally and cannot stop at either of the two external vertices in this specific diagram, they have to form a closed loop. Analyzing the external vertices in this manner, we can thus state that some index combinations will necessarily result in time-arrow loops and are, hence, forbidden. For the case of interest here, the two lower diagrams of Fig. 1 are of this type and vanish.
${ }^{1}$ J. Gabelli, G. Feve, J. M. Berroir, B. Placais, A. Cavanna, B. Etienne, Y. Jin, and D. C. Glattli, Science 313, 499 (2006).

${ }^{2}$ M. Büttiker, A. Prêtre, and H. Thomas, Phys. Rev. Lett. 70, 4114 (1993).

${ }^{3}$ M. Büttiker, H. Thomas, and A. Prêtre, Phys. Lett. A 180, 364 (1993).

${ }^{4}$ A. Prêtre, H. Thomas, and M. Büttiker, Phys. Rev. B 54, 8130 (1996).

${ }^{5}$ G. Feve, A. Mahe, J. M. Berroir, T. Kontos, B. Placais, D. C. Glattli, A. Cavanna, B. Etienne, and Y. Jin, Science 316, 1169 (2007).

${ }^{6}$ Y. Meir and N. S. Wingreen, Phys. Rev. Lett. 68, 2512 (1992).

${ }^{7}$ J. Rammer and H. Smith, Rev. Mod. Phys. 58, 323 (1986).
${ }^{8}$ Y. Fu and S. C. Dudley, Phys. Rev. Lett. 70, 65 (1993).

${ }^{9}$ A. P. Jauho, N. S. Wingreen, and Y. Meir, Phys. Rev. B 50, 5528 (1994).

${ }^{10}$ Z. Feng, J. Maciejko, J. Wang, and H. Guo, Phys. Rev. B 77, 075302 (2008).

${ }^{11}$ X. Q. Li and Z. B. Su, Phys. Rev. B 54, 10807 (1996).

${ }^{12}$ J. Fransson, O. Eriksson, and I. Sandalov, Phys. Rev. B 66, 195319 (2002).

${ }^{13}$ Y. L. U. Gavish and Y. Imry, in Electronic Correlations: From Meso- to Nano-Physics, edited by G. M. T. Martin and J. T. T. Van, Proceedings of the XXXVI Rencontres de Moriond (EDP Sciences, Les Ulis, 2001), p. 243.

${ }^{14}$ E. A. Rothstein, O. Entin-Wohlman, and A. Aharony, Phys. Rev. 
B 79, 075307 (2009).

${ }^{15}$ O. L. Bo and Y. Galperin, J. Phys.: Condens. Matter 8, 3033 (1996).

${ }^{16}$ J. X. Zhu and A. V. Balatsky, Phys. Rev. B 67, 165326 (2003).

${ }^{17}$ M. Galperin, A. Nitzan, and M. A. Ratner, Phys. Rev. B 74, 075326 (2006).

${ }^{18}$ I. Safi and H. J. Schulz, Phys. Rev. B 52, R17040 (1995).

${ }^{19}$ C. Mora and K. Le Hur, arXiv:0911.4908 (unpublished).

${ }^{20} \mathrm{C}$. Moca, I. Weymann, and G. Zarand, arXiv:0907.0475 (unpublished).

${ }^{21}$ R. Avriller and A. Levy Yeyati, Phys. Rev. B 80, 041309(R) (2009).

${ }^{22}$ F. Haupt, T. Novotný, and W. Belzig, Phys. Rev. Lett. 103,
136601 (2009).

${ }^{23}$ T. L. Schmidt and A. Komnik, Phys. Rev. B 80, 041307(R) (2009).

${ }^{24}$ S. E. Nigg and M. Büttiker, Phys. Rev. B 77, 085312 (2008).

${ }^{25}$ Note that the dot-electron lines include the effects of tunneling excursions to the leads.

${ }^{26}$ As a special case, we have to account for the possibility of directly connecting the lead electron lines from the two external vertices without the inclusion of a dot-electron Green's function. This extra "direct" contribution is only possible when the two vertices involve lead-electron operators from the same lead, i.e., for the correlator $K_{\alpha \alpha}$. 